Prix Nobel de Médecine 2013

James Rothman, Randy Schekman

et Thomas Südhof

\section{Une récompense pour la découverte des acteurs et des mécanismes moléculaires fondamentaux du trafic vésiculaire intracellulaire}

Thierry Galli ${ }^{1,2^{*}}$, Aurélia Kuster ${ }^{1,2}$, David Tareste ${ }^{1,2}$

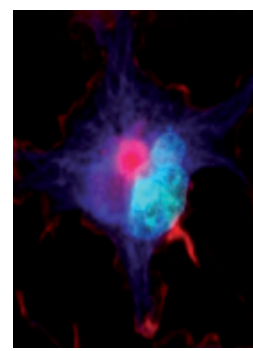

Randy Schekman (University of California, Berkeley), né à Saint Paul (Minnesota) en 1948, est professeur de biologie moléculaire et cellulaire à l'Université de Californie à Berkeley et ancien rédacteur en chef des Comptes rendus de l'Académie des sciences américaines (PNAS). II a soutenu sa thèse en 1975 à l'université de Stanford (Californie). En 2002, il a reçu le prix Lasker en compagnie de James Rothman, un de ses co-lauréats.

Thomas Südhof (Stanford University, Palo Alto), né en 1955 à Göttingen en Allemagne, est titulaire d'un doctorat de neurochimie de l'université de sa ville natale. En 1983, il rejoint les États-Unis, d'abord à Dallas, au centre médical de la Southwestern university (Texas), avant de devenir en 2008 professeur de médecine à l'école de médecine de Stanford (Californie). Membre de l'Académie américaine des sciences et de l'Institut de médecine, Thomas Südhof a reçu en septembre 2013 le prestigieux prix Lasker. <
La cellule eucaryote est délimitée par une bicouche lipidique, la membrane plasmique, et composée de différents compartiments membranaires intracellulaires. Pour maintenir cette organisation et conserver l'intégrité structurelle et fonctionnelle des différents compartiments, la cellule se repose sur un ensemble d'événements de trafic membranaire dont les véhicules sont des vésicules lipidiques. Les échanges de matière et d'information assurés par le trafic vésiculaire sont essentiels à la vie cellulaire eucaryote puisqu'ils permettent aux compartiments de communiquer les uns avec les autres mais également à la cellule de communiquer avec son milieu extérieur. En perpétuel remaniement, les compartiments membranaires apparaissent stables grâce à un équilibre dynamique entre flux entrants et sortants, et présentent un état stationnaire de non-équilibre fluctuant typique des systèmes vivants [1]. Le prix Nobel de Médecine ou Physiologie 2013 récompense Randy Schekman, James Rothman et Thomas Südhof, trois chercheurs ayant apporté des contributions majeures à la compréhension des mécanismes moléculaires du transport vésiculaire intracellulaire (Figure 1). II se situe dans la droite ligne du prix Nobel de 1974 attribué à Albert Claude, Christian de Duve et George Palade pour leur découverte de la compartimentation cellulaire en structures 
membranaires et de la vectorialité du trafic des protéines de sécrétion qui partent du réticulum endoplasmique, passent par l'appareil de Golgi, puis sont stockées dans des vésicules de sécrétion, qui les acheminent vers la membrane plasmique.

\section{Des acteurs moléculaires conservés}

R. Schekman et ses collègues ont choisi une approche génétique pour identifier les acteurs moléculaires clefs du trafic vésiculaire en utilisant comme modèle la levure Saccharomyces cerevisiae. Ils ont profité du fait que des levures mutantes présentant des défauts de sécrétion accumulent des protéines et des lipides nouvellement synthétisés tandis que leur membrane plasmique cesse de croître, induisant ainsi une augmentation de la densité cellulaire. Cela leur a permis d'isoler simplement, par centrifugation, les levures mutantes denses (donc avec des défauts de sécrétion et de croissance) puis d'identifier les gènes impliqués. Cette approche a conduit à l'identification de 23 produits de gènes, appelés Secl-23, et impliqués dans différentes étapes de la voie de sécrétion [2]. Les mutants Secl7 et Secl 8 présentaient notamment un « embouteillage vésiculaire », suggérant un rôle dans la fusion membranaire [3]. J. Rothman et ses collègues ont utilisé le fait que les protéines subissent des glycosylations bien spécifiques le long de la voie de sécrétion, permettant ainsi de suivre leur localisation, pour reconstituer in vitro le transport intra-Golgi à partir d'extraits de cellules mammifères. Cette approche biochimique a conduit à la découverte de nombreuses molécules essentielles au trafic membranaire. La première molécule identifiée a été appelée NSF (pour $N$-ethylmaleimide sensitive factor) car elle était sensible à un agent alkylant, le $\mathrm{N}$-éthylmaléimide. Or, il s'est avéré que NSF était l'homologue mammifère du produit du gène Secl8 identifié dans le crible de R. Schekman [4], mettant ainsi en évidence une convergence majeure entre les travaux des deux chercheurs. Cette convergence avait une implication fondamentale car elle indiquait la conservation des acteurs moléculaires du trafic membranaire de la levure aux mammifères. J. Rothman et ses collaborateurs ont alors déroulé la pelote avec NSF comme point de départ, et ont ensuite identifié la protéine a-SNAP (soluble NSF attachment protein) qui permet la liaison de NSF aux membranes golgiennes. Ils ont de plus montré que le cytosol du mutant Secl7 ne permettait pas la liaison de NSF ni le transport vésiculaire, et que ces défauts pouvaient être compensés par l'ajout de a-SNAP purifiée, suggérant ainsi l'équivalence fonctionnelle entre $\mathrm{Sec} 17$ et a-SNAP [5]. Comme NSF et SNAP étaient impliqués dans toutes les étapes de la voie de sécrétion, d'autres facteurs devaient permettre aux vésicules d'atteindre la bonne membrane cible et fusionner. R. Schekman et J. Rothman émirent alors l'hypothèse selon laquelle SNAP et NSF faisaient partie d'un complexe multi-protéique membranaire dont les sous-unités apportant la spécificité aux événements de fusion devaient être identifiées [6].

\section{L'hypothèse SNARE}

J. Rothman et ses collaborateurs ont alors pensé que les protéines de ce complexe seraient d'autant plus abondantes, et donc faciles à pêcher, que le trafic vésiculaire est actif ; ils se sont donc tournés vers le système nerveux. En utilisant SNAP et NSF comme appât, ils ont isolés trois protéines à partir d'un extrait de cerveau bovin, qu'ils ont appelées SNARE (pour SNAP receptors) [7]. Ces trois protéines avaient déjà été identifiées, entre autres par De Camilli, Jahn, Scheller et Südhof, comme des protéines abondantes de la terminaison présynaptique : Synaptobrevine 2/VAMP2, Syntaxine 1 et SNAP-25 [8-10], mais leur fonction exacte était inconnue. Comme Synaptobrevine est localisée dans les vésicules synaptiques, tandis que Syntaxine réside dans la membrane plasmique présynaptique, J. Rothman a proposé la fameuse «hypothèse SNARE » selon laquelle une vésicule de transport s'accroche et fusionne avec une membrane cible lorsqu'une protéine v-SNARE de la vésicule interagit avec une protéine $t-S N A R E$ de la membrane cible (target) (Figure 1). Cet article reste un cas très particulier dans I'histoire récente des publications en «article » à Nature puisqu'il a pour donnée principale le puits d'un gel d'électrophorèse coloré au bleu de Coomassie avec identification des protéines visualisées. Un rôle essentiel des SNARE dans la libération des neurotransmetteurs a été proposé à peu près au même moment par De Camilli, Jahn, Montecucco, Niemann et Südhof suite à la caractérisation du mécanisme moléculaire d'action des neurotoxines produites par les bactéries Clostridium botulinum et tetani : ces neurotoxines inhibent la transmission synaptique en clivant les protéines Synaptobrevine, SNAP-25 ou Syntaxine [11-13]. Le principe a ensuite été généralisé à la sécrétion des cellules non neuronales [14] et à tous les phénomènes de transport entre compartiments membranaires des cellules eucaryotes [15]. Chaque événement de fusion intracellulaire impliquerait donc une paire originale v-SNARE/t-SNARE dont l'assemblage donnerait la spécificité de l'amarrage de la vésicule à la membrane cible. Les prédictions formulées en 1993 ne sont pas valables dans tous les détails, notamment concernant la spécificité des combinaisons $v$-SNARE/t-SNARE, mais l'hypothèse émise par J. Rothman a fourni un cadre conceptuel qui a stimulé et guidé vingt ans de recherche visant à comprendre les mécanismes moléculaires du trafic membranaire [16, 17]. Les protéines SNARE sont en effet nécessaires et suffisantes pour fusionner des membranes artificielles in vitro [18] et des cellules modifiées exprimant des t-SNARE ou des v-SNARE sur leur membrane plasmique [19]. Elles s'assemblent à l'image d'une fermeture Éclair et fournissent l'énergie requise pour fusionner les bicouches lipidiques [20-22]. Enfin, dans des mutants de Synaptobrevine ou SNAP-25 chez le nématode, la mouche ou la souris, la transmission synaptique est massivement abrogée [23-26].

\section{Une mécanique bien huilée}

J. Rothman et R. Schekman ont donc identifié les acteurs moléculaires clés et les mécanismes fondamen- 


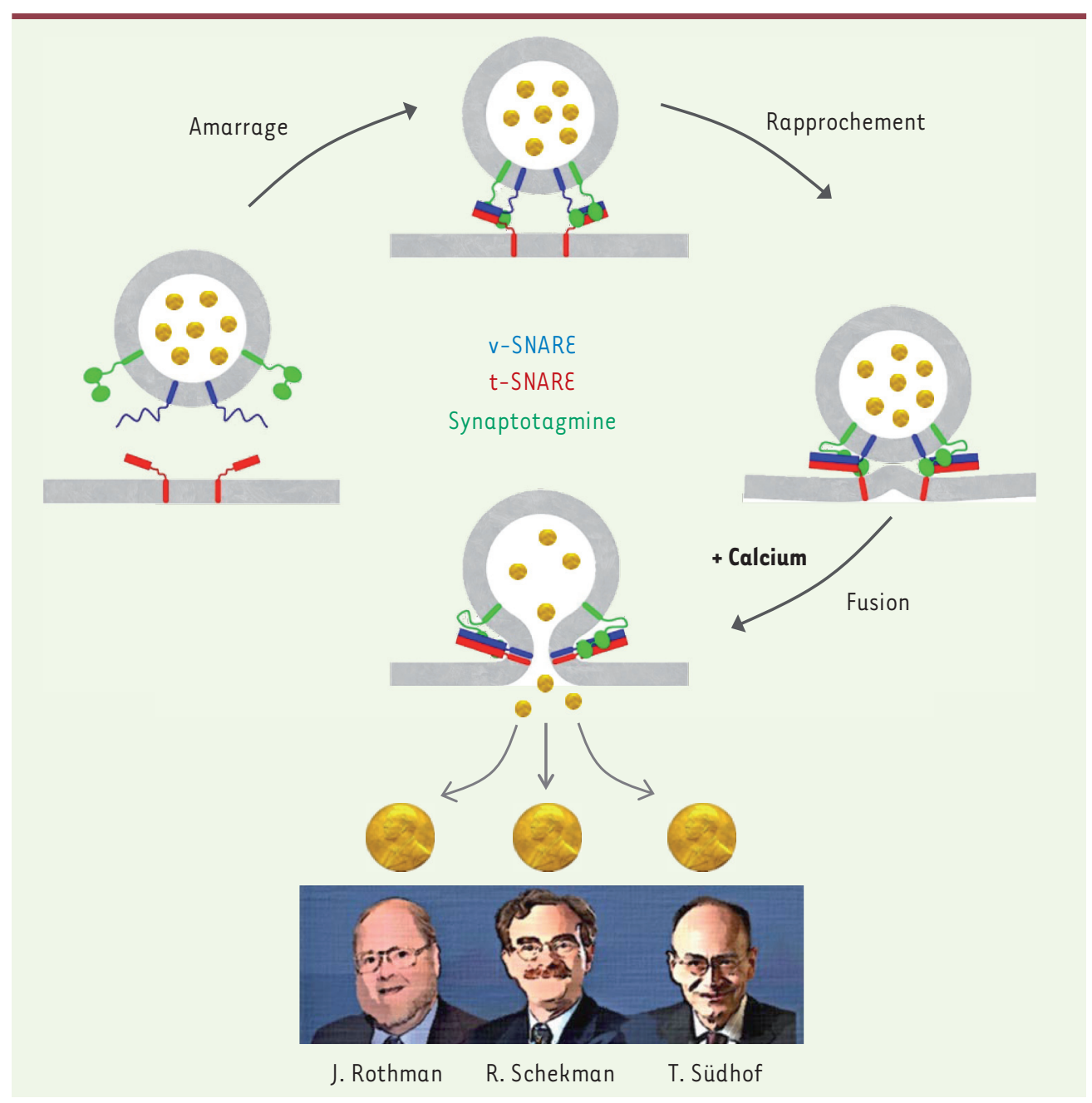

Figure 1. Les découvertes de J. Rothman, R. Schekman et T. Südhof ont permis d'élucider les mécanismes moléculaires qui orchestrent le trafic vésiculaire. Une vésicule de transport fusionne avec une membrane cible lorsque la protéine v-SNARE de la vésicule s'assemble avec sa partenaire t-SNARE de la membrane cible. Dans le cas des événements de fusion dépendants du calcium (par exemple la neurotransmission), la fusion est déclenchée lorsque la protéine Synaptotagmine lie le calcium entraînant une série d'interactions avec les SNAREs et les membranes, qui conduisent à la fusion.

ainsi compte de l'extrême précision et rapidité de la transmission synaptique in vivo [28-30]. Complexine et Synaptotagmine constitueraient donc respectivement le frein

taux du transport et de la fusion vésiculaire. Cependant, il manquait des éléments essentiels au tableau, notamment pour rendre compte de la sensibilité au calcium de la fusion des vésicules synaptiques qui leur permet de libérer les neurotransmetteurs au bon moment et en un temps record (inférieur à la milliseconde). T. Sudhöf et ses collaborateurs ont identifié deux protéines de la régulation temporelle de la machinerie de fusion membranaire SNARE : Synaptotagmine et Complexine. Synaptotagmine lie les membranes et le complexe SNARE de manière dépendante du calcium, avec une faible affinité pour cet ion. Cela apparaît essentiel dans la démonstration du rôle de Synaptotagmine comme senseur du calcium intracellulaire car une concentration très élevée en calcium, telle qu'on peut la mesurer à proximité immédiate des canaux calcium dépendants du voltage, entraîne la libération des neurotransmetteurs. Dans une expérience très élégante, T. Südhof a généré une souris dont la Synaptotagmine a une affinité altérée pour le calcium entraînant une transmission synaptique moins dépendante du calcium [27]. Les travaux de T. Sudhöf et J. Rothman ont montré que Complexine lie le complexe SNARE et arrête la réaction dans un état proche de la fusion, qui n'attend plus que l'entrée du calcium dans la terminaison nerveuse (et sa liaison à Synaptotagmine) pour redémarrer à une vitesse fulgurante (Figure 1), rendant et l'accélérateur de la machinerie de fusion synaptique. T. Südhof et ses collaborateurs ont appliqué une approche systématique d'identification des protéines impliquées dans la physiologie de la synapse neuronale, et de caractérisation de leur fonction dans des mutants d'invalidation du gène chez la souris. On peut également citer les travaux sur Munc18-1, une protéine mammifère homologue de la protéine de levure Secl (la première identifiée dans le test de Schekman) dont la suppression chez la souris conduit à une perte totale de neurotransmission [31]. II se trouve que Munc18-1 est un régulateur essentiel de l'activité de la molécule Syntaxine 1 [32] et des travaux récents de J. Rothman ont montré que Muncl8-1 stimule exclusivement la fusion induite par le complexe v-SNARE/t-SNARE synaptique, contribuant ainsi à la régulation spatiale des événements de fusion [33]. Les connaissances actuelles du mode d'action de ces différentes protéines qui règlent les rouages de la machinerie SNARE sont présentées dans une revue que Südhof a récemment coécrite avec Rothman [34]. L'approche incroyablement 
productive de T. Südhof a généré des modèles expérimentaux utilisés par de nombreuses équipes de recherche pour comprendre le rôle des protéines synaptiques et la fonction des phénomènes sécrétoires notamment au cours du développement [35-37].

La boucle est ainsi bouclée, de $\mathrm{Secl}$ chez la levure à son homologue Munc18-1 chez l'homme. L'importance biomédicale des travaux récompensés par le prix Nobel 2013 est presque secondaire par rapport aux avancées conceptuelles que R. Schekman, J. Rothman et T. Sudhöf ont réalisées quarante ans après le prix Nobel de Claude, de Duve et Palade. Leurs découvertes des acteurs et des mécanismes moléculaires de la fusion membranaire sont pertinentes pour des organismes aussi distants que l'homme et la levure, et s'appliquent à de nombreux phénomènes physiologiques fondamentaux comme le transport des molécules au sein des cellules, la communication neuronale, la sécrétion hormonale et la réponse immunitaire. Le système immunitaire apparaît particulièrement sensible aux perturbations du trafic vésiculaire comme le montrent les mutations des gènes SNARE ou MUNC18 chez des patients immunodéficients et des souris mutantes [38-42]. Une nouvelle page s'ouvre maintenant. L'utilisation d'approches multidisciplinaires et intégrées combinant la génétique, la physiologie, la biologie cellulaire et la biophysique devrait encore permettre des avancées majeures pour comprendre le détail fin des mécanismes moléculaires du trafic vésiculaire et l'incroyable dynamique qui parcourt à chaque instant nos cellules. $\diamond$

Nobel Prize in Physiology and Medicine 2013 - An award

for the discovery of the actors and fundamental molecular mechanisms of intracellular vesicle trafficking

\section{LIENS D'INTÉRÊT}

Les auteurs déclarent n'avoir aucun lien d'intérêt concernant les données publiées dans cet article.

\section{RÉFÉRENCES}

1. Prigogine I. La thermodynamique de la vie. La Recherche $2000 ; 99: 38$.

2. Novick P, Field C, Schekman R. Identification of 23 complementation groups required for posttranslational events in the yeast secretory pathway. Cell $1980 ; 21: 205-15$.

3. Kaiser CA, Schekman R. Distinct sets of SEC genes govern transport vesicle formation and fusion early in the secretory pathway. Cell $1990 ; 61: 723-33$.

4. Wilson DW, Wilcox CA, Flynn GC, et al. A fusion protein required for vesicle-mediated transport in both mammalian cells and yeast. Nature 1989 ; 339 : 355-9.

5. Clary DO, Griff IC, Rothman JE. SNAPs, a family of NSF attachment proteins involved in intracellular membrane fusion in animals and yeast. Cell $1990 ; 61: 709-21$.

6. Griff IC, Schekman R, Rothman JE, Kaiser CA. The yeast SEC17 gene product is functionally equivalent to mammalian $\alpha$-SNAP protein. J Cell Biol $1992 ; 267: 12106-15$.

7. Söllner T, Whiteheart SW, Brunner $M$, et al. SNAP receptors implicated in vesicle targeting and fusion. Nature $1993 ; 362: 318-24$.

8. Baumert M, Maycox PR, Navone F, et al. Synaptobrevin: an integral membrane protein of 18,000 daltons present in small synaptic vesicle of rat brain. EMBO J $1989 ; 8: 379-84$.

9. Bennett MK, Calakos N, Scheller RH. Syntaxin: a synaptic protein implicated in docking of synaptic vesicles at presynaptic active zones. Science $1992 ; 257: 255-9$.

10. Oyler GA, Polli JW, Higgins GA, et al. Distribution and expression of SNAP-25 immunoreactivity in rat brain, rat PC-12 cells and human SMS-KCNR neuroblastoma cells. Dev Brain Res 1992; 65 : 133-46.

11. Schiavo G, Benfenati F, Poulain B, et al. Tetanus and botulinum-B neurotoxins block neurotransmitter release by proteolytic cleavage of synaptobrevin. Nature $1992 ; 359: 832-5$.

12. Blasi J, Chapman $\varepsilon R$, Link $\varepsilon$, et al. Botulinum neurotoxin $A$ selectively cleaves the synaptic protein SNAP-25. Nature $1993 ; 365: 160-3$.

13. Blasi J, Chapman $\varepsilon R$, Yamasaki $S$, et al. Botulinum neurotoxin $\mathrm{Cl}$ blocks neurotransmitter release by means of cleaving HPC-1/syntaxin. EMBO J 1993 ; 12: 4821-8.
14. Galli T, Chilcote T, Mundigl 0, et al. Tetanus toxin-mediated cleavage of cellubrevin impairs exocytosis of transferrin receptor-containing vesicles in CHO cells. J Cell Biol $1994 ; 125$ : 1015-24.

15. Ferro-Novick S, Jahn R. Vesicle fusion from yeast to man. Nature 1994 ; 370 : 191-3.

16. Galli T, Martinez Arca S, Paumet F. Mécanisme de la fusion membranaire. Med Sci (Paris) $2002 ; 18: 1113-9$.

17. Seagar M, Quetglas S, Iborra C, Leveque C. Le complexe SNARE au cœur de la fusion membranaire. Med Sci (Paris) $2001 ; 17: 669-74$.

18. Weber T, Zemelman BV, McNew JA, et al. SNAREpins: minimal machinery for membrane fusion. Cell $1998 ; 92: 759-72$.

19. Hu C, Ahmed M, Melia TJ, et al. Fusion of cells by flipped SNAREs. Science $2003 ; 300: 1745-9$

20. Sutton RB, Fasshauer D, Reinhard J, Brunger AT. Crystal structure of a SNARE complex involved in synaptic exocytosis at $2.4 \AA$ resolution. Nature 1998 ; $395:$ 347-53.

21. Li F, Pincet F, Perez $\varepsilon$, et al. Energetics and dynamics of SNAREpin folding across lipid bilayers. Nat Struct Mol Biol 2007 ; 14 : 890-6.

22. Tareste $D$. Énergie libérée par la machinerie de fusion SNAREpin. Med Sci (Paris) $2008 ; 24: 142-3$

23. Nonet ML, Saifee 0 , Zhao HJ, et al. Synaptic transmission deficits in Caenorhabditis elegans synaptobrevin mutants. J Neurosci 1998; $18: 70-80$.

24. Deitcher DL, Ueda A, Stewart BA, et al. Distinct requirements for evoked and spontaneous release of neurotransmitter are revealed by mutations in the Drosophila gene neuronal-synaptobrevin. J Neurosci 1998; 18 : 2028-39.

25. Schoch S, Deak F, Konigstorfer A, et al. SNARE function analyzed in synaptobrevin/VAMP knockout mice. Science 2001 ; 294 : 1117-22.

26. Washbourne P, Thompson PM, Carta M, et al. Genetic ablation of the t-SNARE SNAP-25 distinguishes mechanisms of neuroexocytosis. Nat Neurosci 2002 . $5: 19-26$.

27. FernandezChacon R, Konigstorfer A, Gerber SH, et al. Synaptotagmin I functions as a calcium regulator of release probability. Nature $2001 ; 410$ : 41-9.

28. McMahon HT, Missler M, Li C, Südhof TC. Complexins: cytosolic proteins that regulate SNAP receptor function. Cell $1995 ; 83: 111-9$.

29. Tang J, Maximov A, Shin $\mathrm{OH}$, et al. A complexin/synaptotagmin 1 switch controls fast synaptic vesicle exocytosis. Cell $2006 ; 126: 1175-87$.

30. Giraudo CG, Eng WS, Melia TJ, Rothman JE. A clamping mechanism involved in SNARE-dependent exocytosis. Science 2006 ; 313 : 676-80.

31. Verhage M, Maia AS, Plomp JJ, et al. Synaptic assembly of the brain in the absence of neurotransmitter secretion. Science 2000 ; 287 : 864-9.

32. Hata $Y$, Slaughter CA, Sudhof TC. Synaptic vesicle fusion complex contains unc-18 homologue bound to syntaxin. Nature 1993 ; 366 : 347-51.

33. Shen J, Tareste D, Paumet F, et al. Selective activation of cognate SNAREpins by Secl/Munc18 proteins. Cell $2007 ; 128: 183-95$.

34. Sudhof TC, Rothman JE. Membrane fusion: grappling with SNARE and SM proteins. Science $2009 ; 323: 474-7$.

35. Nicol X, Voyatzis S, Muzerelle A, et al. cAMP oscillations and retinal activity are permissive for ephrin signaling during the establishment of the retinotopic map. Nat Neurosci $2007 ; 10: 340-7$.

36. Zylbersztejn K, Petkovic M, Burgo A, et al. The vesicular SNARE Synaptobrevin is required for Semaphorin 3A axonal repulsion. J Cell Biol 2012 ; 196: 37-46.

37. Zylbersztejn K, Galli T. Le trafic membranaire, un nouvel acteur du guidage axonal. Med Sci (Paris) $2012 ; 28: 267-9$.

38. Danielian S, Basile N, Rocco C, et al. Novel syntaxin 11 gene (STX11) mutation in three Argentinean patients with hemophagocytic lymphohistiocytosis. J Clin Immunol 2010 ; 30 : 330-7.

39. Cote M, Menager MM, Burgess A, et al. Munc18-2 deficiency causes familial hemophagocytic lymphohistiocytosis type 5 and impairs cytotoxic granule exocytosis in patient NK cells. J Clin Invest 2009; 119 : 3765-73.

40. Menager MM, Menasche G, Romao M, et al. Secretory cytotoxic granule maturation and exocytosis require the effector protein hMuncl3-4. Nat Immunol $2007 ; 8: 257-67$.

41. Feldmann J, Callebaut I, Raposo G, et al. Munc13-4 is essential for cytolytic granules fusion and is mutated in a form of familial hemophagocytic lymphohistiocytosis (FHL3). Cell 2003 ; 115 : 461-73.

42. Larghi P, Williamson DJ, Carpier JM, et al. VAMP7 controls T cell activation by regulating the recruitment and phosphorylation of vesicular Lat at TCRactivation sites. Nat Immunol $2013 ; 14: 723-31$.

\section{TIRÉS À PART}

T. Galli 